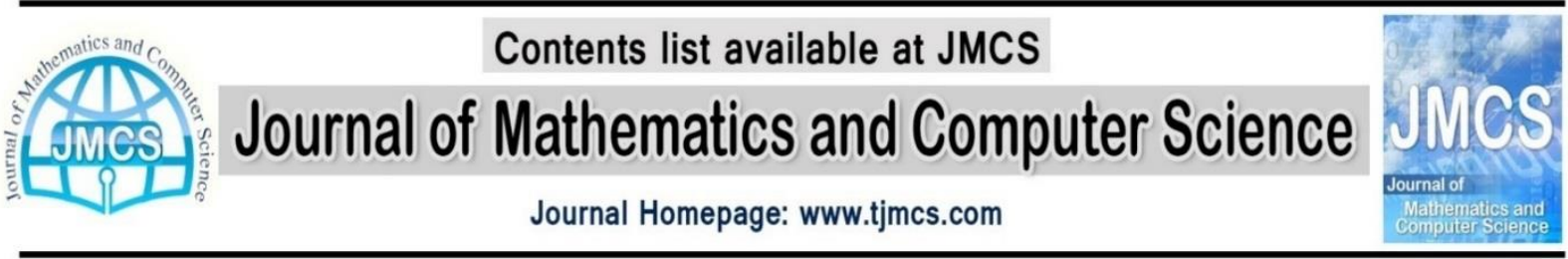

\section{A Nonlinear Dynamic Logistics Model for Disaster Response under Uncertainty}

\author{
M. Khorsi ${ }^{1}$, A. Bozorgi-Amiri ${ }^{2 \star}$, B. Ashjari ${ }^{3}$ \\ ${ }^{1}$ Department of Industrial Engineering, Tafresh University, Tafresh, Iran, \\ malihe.khorsi@gmail.com \\ ${ }^{2}$ Department of Industrial and Systems Engineering, College of Engineering, University of Tehran, Tehran, Iran, \\ alibozorgi@ut.ac.ir \\ ${ }^{3}$ Department of Industrial Engineering, Tafresh University, Tafresh, Iran, \\ ashied@aut.ac.ir
}

Article history:

Received February 2013

Accepted March 2013

Available online April 2013

\begin{abstract}
To save lives and alleviate suffering, the response to emergency must be timely, effective, appropriate, and well organized. Logistics can play a key role. This paper presents a multi-objective dynamic stochastic model for a complex logistical problem in disaster relief operations. Prior to the disaster onset, design decisions including the number and location of local distribution centers needed as well as their inventory levels for each type of emergency supply are made. After the disaster onset, the designed network will use to conduct daily operational decisions over a planning horizon that covers the disaster duration. The first objective function attempts to minimize the sum of the expected value of the total cost of the relief chain; at the same time the model aims to maximize the affected areas' satisfaction levels through minimizing the sum of the maximum shortages in the affected areas. A case study is presented to illustrate the potential applicability of our model for disaster planning for earthquake scenarios in the megacity of Tehran. The results demonstrate the practicability of the proposed multiobjective stochastic model.
\end{abstract}

Keywords: Disaster relief logistics, stochastic programming, Multi-objective optimization.

\section{Introduction}

According to the EM-DAT, from 2000 to 2010 exactly 8351 disasters (e.g., Earthquake) occurred all over the world which, on average, amounts to more than two new disasters every day [1].The worldwide increasing trend in the number of large-scale natural disasters and the number of people reportedly affected, leading to a greater need for efficient disaster management. Quick response to the urgent relief needs right after natural disasters through efficient relief logistics is vital to the alleviation of disaster impact in the affected areas (AAs).Pre-positioning critical items in strategic locations and effective distribution of the items after a disaster

\footnotetext{
* Corresponding author
} 
are considered to be useful tools to a quick response. An evident difficulty in creating an effective prepositioning plan is uncertainty about whether or not natural disasters will occur, and if they do, where and with what magnitude. Our goals in this paper are to determine:

- the optimum number, location and size of relief distribution centers (RDCs) to be used,

- the amount of inventory to be procured at various RDCs in the pre-disaster phase, and,

- The transportation amount to be delivered the commodities to the AAs over a planning horizon under uncertain parameters.

The objective functions of our proposed model are the minimizing of the maximum amount of shortages among the AAs, and the sum of pre- and post-disaster costs. The main contributions of this paper can be summarized as follows:

- Achieving a model which contemplates the different sources of uncertainty.

- Developing a new multi-objective dynamic stochastic model to tackle the disaster relief problem.

- Considering disruption in a facility by a disaster.

- Considering fair distribution to certain affected areas in the relief distribution process.

- Applying the model to a real-world disaster relief chain.

The rest of this paper is organized as follows. In Section 2, the relevant literature is reviewed. In Section 3, the general problem description statement is given and a multi-objective stochastic optimization model is developed to formulate the relief logistics problem. In Section 4, a solution method is presented. Then, the effectiveness of the proposed model is demonstrated by a case analysis and experimental results in Section 5. Our conclusions and future research plans are presented in the final section.

\section{Literature review}

Depending on whether uncertainty is taken into account or no, work reported in the literature of this problem can be classified into two major categories: deterministic or stochastic. In the deterministic case, it is assumed that all problem inputs like demand, supply and costs quantities are known in advance with certainty (e.g. [2], [3], [4]).

The significance of uncertainty has motivated a number of researchers to address stochastic optimization in disaster relief planning involving distribution of emergency relief commodities by probabilistic scenarios representing disasters and their outcomes (see [5] - [9]).

Reference [10] developed a two-stage stochastic optimization model to plan the allocation of budget to acquire and position relief assets. However, they did not consider the possibility of inventory being destroyed. Reference [11] Introduced stochastic optimization model for disaster preparedness and response to assist with decisions for the location and allocation of medical supplies. Reference [12] developed a two-stage, stochastic, mixed-integer program that determined the locations and quantities of various types of emergency commodities; their model also considered the transportation network availability following a disaster. Reference [7] proposed a two-stage stochastic model. The first stage determines the location of RDCs and the required inventory quantities for each type of relief items under storage, and the second stage determines the amount of transportation from distribution centers to AAs.

Though these efforts have provided us different concepts for handling disaster relief operations efficiently, integrating strategic, tactical and operational decisions remains rare in the literature.

We present a new model that integrates location, inventory and allocation decisions. We consider a multicommodity, multi-period, multi-modal transportation under uncertainty. Environmental uncertainty is described by discrete scenarios. The proposed model minimizes the sum of the maximum amount of shortages among the AAs in all periods and sum pre- and post-disaster costs. 


\section{Problem description}

Disaster relief logistics network considered in this paper consists of the set of RDCs and the set of AAs (Figure1). The relief items stored in RDCs, which are located prior to the disaster, are distributed to the demand points. RDCs are large facilities in which all types of relief items are stocked. They are established to satisfy the demand of the relief items in disaster areas. Thus, their locations as well as relief items flowing through them to demand points are related with the amount and site of the needs that occur in a scenario.

(1) The capability of candidate RDCs may be partially disrupted by a disaster through damage to the roads and/or destruction to the facility.

(2) The level of demand for the AA and the cost parameters are uncertain and depend on various factors including the disaster scenario and the impact of the disaster. To represent uncertain parameters, we make use of discrete scenarios from a set $\mathrm{S}$ of possible disaster situations. We assume that the probability distribution of scenarios can be devised by subject matter experts or disaster planners.

(3) Emergency supplies are divided in to two major categories: consumable and non-consumable items. Consumable items are items that are consumed regularly and whose demand occurs periodically over the planning horizon, such as water and food. Non-consumable items are critical items for which the demand occurs once at the beginning of the planning horizon, such as shelters and electricity devices.

(4) The logistics plan involves a planning time horizon consisting of a given number of time periods because it deals with time-variant demand and supply.

(5) An RDC can be opened in only one of three possible configurations with distinct storage capacity (small, medium, or large), subject to the associated setup cost.

(6) Each RDC may be supplied by other RDCs (backup coverage).

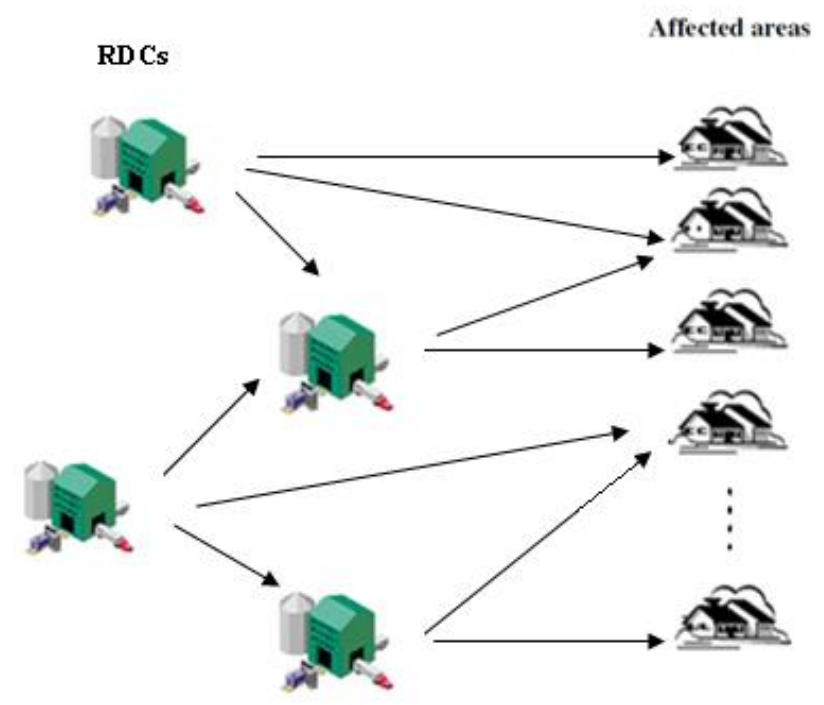

Figure 1: General schema of relief distribution chain

\subsection{Notation, Parameters, and Decision Variables Sets/ indices}

I Set of candidate RDCs indexed by $i \in I$ 
$J \quad$ Set of AAs by disaster indexed by $j \in J$

$S \quad$ Set of possible scenarios indexed by $s \in S$

$C \quad$ Set of commodities indexed by $c \in\{1,2\}$

$V \quad$ Set of transportation modes indexed by $v \in V$

$L \quad$ Set of size of RDCs indexed by $l \in L$

$T \quad$ Set of periods indexed by $t \in T$

\section{Parameters}

$f_{i l} \quad$ Fixed cost for opening a RDC of size $l$ at location $i$

$\varphi_{c i} \quad$ Procuring cost of a unit commodity $c$ in RDC $i$

$h_{c i} \quad$ Inventory holding cost for a unit commodity $c$ at RDC $i$

$h_{c j} \quad$ Penalty of a unit commodity $c$ haven't consumed in end of planning horizon at AA $j$

$\operatorname{cap}_{l c} \quad$ Capacity of RDC of type $l$ for commodity $c$

$p_{s} \quad$ Occurrence probability of scenarios

$\varphi_{\text {cist }} \quad$ Procuring cost of a unit commodity $c$ in RDC $i$ under scenario $s$ in period $t$

$c_{c i j v s t} \quad$ Transportation cost of a unit commodity c from RDC $i$ to AA $j$ under scenario $s$ by mode $v$ at period $t$

$\pi_{c j s t}$ Inventory shortage cost for a unit commodity $c$ to AA $j$ under scenario $\sin$ period $t$

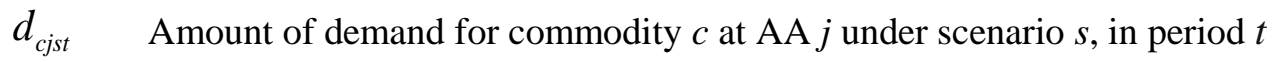

$\rho_{\text {cis }} \quad$ Fraction of stocked material of commodity c at RDC $i$ that remains usable in scenario $s\left(0 \leq \rho_{\text {cis }} \leq 1\right)$

$\alpha_{c j s t} \quad$ Weight factor for commodity $c$ in AA $j$, in scenario $s$ and period $t$

$\beta_{j s t} \quad$ Weight factor for AA $j$ in scenario $s$ and period $t$

\section{Variables}

$Z_{i l} \quad 1$ if RDC with capacity category $l$ is located at candidate RDC $i ; 0$ otherwise

$S_{c i} \quad$ Amount of commodity $c$ stored in RDC $i$

$X_{c i j s t} \quad$ Amount of commodity $c$ transferred from RDC $i$ by mode $v$ to AA $j$ in scenario $s$ and period $t$

$Q_{\text {cist }} \quad$ Amount of commodity $c$ procured for RDC $i$, in scenario $s$ and period $t$

$I_{\text {cist }} \quad$ Amount of inventory held at $\mathrm{RDC} i$ in scenario $s$ and period $t$

$I_{c j s t} \quad$ Amount of inventory held at AA $j$ in scenario $s$ and period $t$

$W_{c j s} \quad$ Amount of commodity $c$ haven't consumed in end of planning horizon at AA $j$ under scenario $s$

$B_{c j s} \quad$ Amount of shortage commodity $c$ in end of planning horizon at AA $j$ under scenario $s$

$b_{c j s t} \quad$ Amount of shortage commodity $c$ at AA $j$ in scenario $s$ and period $t$

\subsection{Mathematical Model}

$\operatorname{Min} Z_{1}=\sum_{s \in S} p_{s} \cdot \sum_{c, t} \operatorname{Max}_{j \in J}\left\{\alpha_{c j s t} \cdot \beta_{j s t} b_{c j s t}\right\}$ 


$$
\begin{aligned}
& \operatorname{Min} Z_{2}=\sum_{i, l} f_{i l} \cdot Z_{i l}+\sum_{c, i} S_{c i} \cdot\left(\varphi_{c i}+h_{c i}\right)+\sum_{s} p_{s}\left(\sum_{c, i, t}\left(\varphi_{c i s t} \cdot Q_{c i s t}+h_{c i} \cdot I_{c i s t}\right)+\sum_{c, i, j, v, t} c_{c i j v s t} \cdot X_{c i j v s t}\right. \\
& \left.+\sum_{c, j, t} \pi_{c j s t} b_{c j s t}+\sum_{c, j} h_{c j} W_{c j s}\right) \\
& \text { s.t. } \\
& \rho_{c i s} . S_{c i}+\sum_{j \neq i \in I, v} X_{c j i v s t}+Q_{c i s t}=\sum_{j \neq i \in I, v} X_{c i j v s t}+I_{c i s t} \quad \forall i \in I, c \in C, s \in S, t=1 \\
& I_{c i s(t-1)}+\sum_{j \neq i \in I, v} X_{c j i v s t}+Q_{c i s t}=\sum_{j \neq i \in I, v} X_{c i j s t}+I_{c i s t} \quad \forall i \in I, c \in C, s \in S, t>1 \\
& I_{c i s(t-1)}+\sum_{v, l} X_{c i i v s t} . Z_{i l}+\left(\sum_{j \neq i \in I, v} X_{c j i v s t}\right)\left(1-\sum_{l} Z_{i l}\right)-d_{c i s t}-b_{c i s(t-1)}=I_{c i s t}-b_{c i s t} \quad \forall i \in J, c=2, s \in S, t \in T \\
& I_{c i s(t-1)}+\sum_{v, l} X_{\text {ciivst }} . Z_{i l}+\left(\sum_{j \neq i \in I, v} X_{c j i v s t}\right)\left(1-\sum_{l} Z_{i l}\right)-d_{c i s t}=I_{c i s t}-b_{c i s t} \quad \forall i \in J, c=1, s \in S, t \in T \\
& v_{c} S_{c i} \leq \sum_{l} \operatorname{cap}_{l c} Z_{i l} \quad \forall i \in I, c \in C \\
& v_{c} \cdot\left(Q_{c i s t}+I_{c i s(t-1)}\right) \leq \sum_{l} \operatorname{cap}_{l c} Z_{i l} \quad \forall i \in I, c \in C, s \in S, t \\
& X_{c i j v s t} \leq M\left(\sum_{l} Z_{j l}+d_{c j s t}\right) \quad \forall i \in I, j \in J, v \in V, s \in S, \\
& c \in C, t \in T \\
& \sum_{j} X_{c i j v s t} \leq M \cdot \sum_{l} Z_{i l} \\
& X_{c j j v t} \leq M \cdot d_{c j s t} \cdot \sum_{l} Z_{j l} \\
& \forall j \in J, c \in C, v \in V, t \in T, s \in S \\
& \forall j \in J, c \in C, v \in V, t \in T, s \in S \\
& \sum_{t}\left(\sum_{v, l} X_{c i i v s t} \cdot Z_{i l}+\left(\sum_{j \neq i \in I, v, l} X_{c j i v s t}\right)\left(1-Z_{i l}\right)-d_{c i s t}\right)=W_{c i s}-B_{c i s} \quad \forall c \in C, i \in J, s \in S \\
& \sum_{l} Z_{i l} \leq 1 \\
& \forall i \in I \\
& Z_{i l} \in\{0,1\} \\
& \forall i \in I \\
& S_{c i}, Q_{c i s t}, X_{c i j v s t}, I_{c i s t}, I_{c j s t}, W_{c j s}, B_{c j s}, b_{c j s t} \geq 0 \\
& \forall i \in I, j \in J, v \in V, s \in S, c \in C \\
& , t \in T, l \in L
\end{aligned}
$$

The first objective function minimizes the maximum total amount of weighted unsatisfied demand in demand points. The second objective function minimizes the expected total cost. The costs include the preparedness phase cost (associated with setup, procurement and holding) and the response phase cost (associated with transportation, inventory holding, and shortage).Constraint (3) is a control balance equation for $t=1$ and each RDC. Similar to constraint (3), constraint (4) is a control balance equation for $t>1$. Constraints (5) and (6) are inventory balance equation for AAs. constraints (5) is written for non-consumable items while unsatisfied (backordered) demand is considered, but constraints (6) is written for consumable items and unsatisfied demand is not considered. The capacity limits of RDCs are represented by (7) and (8). Constraint (9) guarantees that a RDC could transfer commodity to other nodes only if there exist either another RDC or an AA on that node. Constraint (10) prevents RDCs from transferring commodity to demand points where no RDC has been opened. Constraint (11) indicates that an RDC could transfer commodity to its own area only if its demand points are affected. Constraint (12) is a control balance equation for each AA. Constraint (13) 
prevents more than one RDC from being placed at any nodes. Finally, feasible regions for variables are enforced by constraints (14) and (15).

\subsection{Linear Model}

As can be observed first objective function and constraints (5), (6) and (12) conclude non-linear terms that linearization as follows: The linear equivalent equations for first objective function could be rewritten with the help of an auxiliary variable $B_{c s t} \geq 0$ as follows:

$$
\begin{array}{ll}
\operatorname{MinZ}_{1}=\sum_{s} p_{s} \cdot \sum_{c, t} B_{c s t} & \\
\text { s.t. } B_{c s t} \geq \alpha_{c j s t} \cdot \beta_{j s t} \cdot b_{c j s t} & \forall c \in C, j \in J, s \in S, t \in T \\
B_{c s t} \geq 0 & \forall c \in C, s \in S, t \in T
\end{array}
$$

Constraint (5) could be transformed to linear terms with the help of integer variables as follows:

$$
\begin{array}{ll}
I_{c i s(t-1)}+G 1_{c i s t}+G 2_{c i s t}-d_{c i s t}-b_{c i s(t-1)}=I_{c i s t}-b_{c i s t} & \\
G 1_{c i s t} \leq \sum_{v} X_{c i i v s t} & \forall i \in J, c=2, s \in S, t \in T \\
G 1_{c i s t} \leq M \cdot \sum_{l} Z_{i l} & \forall i \in J, c=2, s \in S, t \in T \\
G 1_{c i s t} \geq \sum_{v} X_{c i i v s t}-M\left(1-\sum_{l} Z_{i l}\right) & \forall i \in J, c=2, s \in S, t \in T \\
G 2_{\text {cist }} \leq \sum_{j \neq i \in I, v} X_{c j i v s t} & \forall i \in J, c=2, s \in S, t \in T \\
G 2_{c i s t} \leq M\left(1-\sum_{l} Z_{i l}\right) & \forall i \in J, c=2, s \in S, t \in T \\
G 2_{c i s t} \geq \sum_{j \neq i \in I, v} X_{c j i v s t}-M \sum_{l} Z_{i l} & \forall i \in J, c=2, s \in S, t \in T
\end{array}
$$

\section{Solution Procedure}

To solve multi-objective optimization problems, three major methods are known: The a priori methods, the a posteriori method and the interactive methods [13].In the a priori method, the decision maker expresses his/her preferences before the solution process, and the multi-objective optimization problem is transformed into a single objective problem. Subsequently, a classical single-objective optimization algorithm is used to find the optimum. The a priori methods can create a representative subset of the Pareto set which in most cases is adequate. The a posteriori method is in the basis of optimizing all objective functions, simultaneously. In this method, first the efficient solutions of the problem (Pareto set) are generated. Then, at the end of the search process, the decision maker involves, in order to select among Pareto set, the most preferred one. In the last method (the interactive methods), the phase in which the decision maker involves in the decision-making process expressing his/her preferences are interchanged with phase of calculation and the process usually converges, after a few iterations, to the most preferred solution. The decision maker successively drives the search with his answers towards the most preferred solution. In this paper, we applied $\varepsilon$-constraint method. In this method, one of the objective functions is selected as the main objective to be optimized, and other objective functions are transformed into constraints by considering an upper bound for each of them. The problem is stated as follows:

$$
\begin{aligned}
& \operatorname{Min} Z_{j}(x) \\
& \text { s.t. } Z_{k}(x) \leq \varepsilon_{k} \quad \forall k \neq j ; x \in X
\end{aligned}
$$




\section{Case Study}

Tehran, the capital of Iran with a population of more than 12 million, is built on a network of faults with high risk to earthquake. According to the earthquake scenarios developed under the JICA-CEST project, Tehran has high seismic potential with many active faults, the most significant are the Mosha fault (with about $68 \mathrm{~km}$ length), Rey fault (with about $26 \mathrm{~km}$ length) and North of Tehran fault (with about $58 \mathrm{~km}$ length)[14].Four earthquake model scenarios were developed based on active faults: (1) the Ray Fault (RF); (2) the Mosha Fault (MF); (3) the North Tehran Fault (NTF); and (4) a floating earthquake not identifiably linked to a particular fault, namely, Floating Fault (FF).We assume the relative probabilities of MF, NTF, RF and FF earthquakes are $0.158,0.352,0.412$, and 0.079 , respectively.

We consider 22 municipal districts of Tehran, which potentially are at risk of earthquake and 22point with low vulnerability as candidate areas for the construction of RDCs were considered (Figure 2).Emergency relief items categorize into two main groups: consumable item e.g. food and water and non-consumable items e.g. shelter. Each consumable item consists of 2 MREs and 3 liter water per person per day. Each shelter can be consumed for 3 people. The various parameters listed in Table 1, 2 and 3.

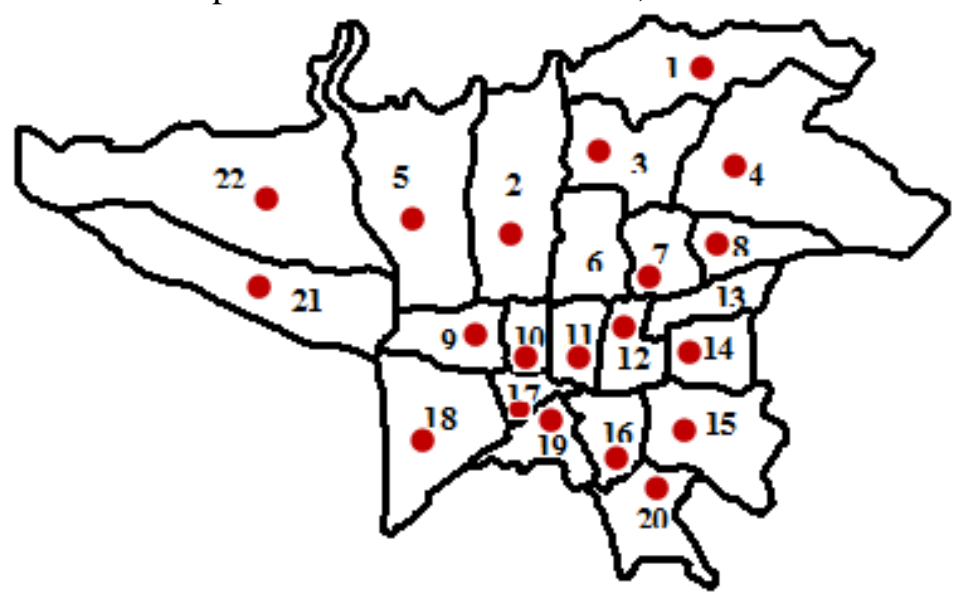

Figure 2. Map of case study: 22 potential AAs and 10 potential RDCs Potential RDC

Table 1: Facility setup cost

\begin{tabular}{cccc}
\hline \multirow{2}{*}{ Size } & $f_{j l}\left(10^{3} \$\right)$ & \multicolumn{2}{c}{$\operatorname{cap}_{l c}\left(10^{3} m^{3}\right)$} \\
\cline { 3 - 4 } & & Con & Non-Con \\
\hline Small & 5000 & 21 & 9 \\
\hline Medium & 8000 & 33.6 & 14.4 \\
\hline Large & 12000 & 50.4 & 21.6 \\
\hline
\end{tabular}

Table2: Unit procurement cost, transportation cost and commodity volume

\begin{tabular}{cccc}
\hline Commodity & $\varphi_{c i}$ (\$/unit) & $v_{c}$ (m3/unit) & Transport. (\$/unit-km) \\
\hline Con & 2.5 & 0.065 & 0.75 \\
\hline Non-Con & 20 & 0.12 & 1.8 \\
\hline
\end{tabular}

The planning horizon is set to 3 days. The penalty cost for unmet demand is estimated to be 20,15 and 10 times, respectively for first, second and third days the post-disaster procurement cost of the corresponding commodity, and the holding cost is estimated according to the current procurement cost. For this problem, only one transportation mode is used which is trucking. Demand for relief items at each demand point for a given scenario are estimated on the basis of the population density multiplied by the vulnerability probability of the demand points. 
Table 3: Demand of consumable commodity $\left(10^{3}\right.$ units $)$

\begin{tabular}{cccccccccc}
\hline \multicolumn{9}{c}{ Faults model } & \multicolumn{3}{c}{ Faults model } \\
\hline DPs & RF & NTF & MF & FF & DPs & RF & NTF & MF & FF \\
\hline 1 & 2719 & 14032 & 1174 & 9581 & 12 & 37058 & 7722 & 3095 & 27264 \\
\hline 2 & 8812 & 11914 & 627 & 16138 & 13 & 10312 & 2614 & 599 & 9551 \\
\hline 3 & 5187 & 10290 & 944 & 11902 & 14 & 22968 & 4191 & 1265 & 18495 \\
\hline 4 & 6777 & 15277 & 2444 & 21804 & 15 & 50973 & 7177 & 1841 & 27765 \\
\hline 5 & 5768 & 12217 & 313 & 12973 & 16 & 29732 & 4213 & 1140 & 17507 \\
\hline 6 & 6517 & 3144 & 570 & 6828 & 17 & 28547 & 3433 & 935 & 15955 \\
\hline 7 & 12817 & 4337 & 831 & 10902 & 18 & 24546 & 2622 & 631 & 13052 \\
\hline 8 & 14610 & 4750 & 786 & 12173 & 19 & 16472 & 1466 & 131 & 6512 \\
\hline 9 & 9755 & 1880 & 158 & 8000 & 20 & 30188 & 2797 & 584 & 14207 \\
\hline 10 & 21983 & 3701 & 373 & 15778 & 21 & 4776 & 1761 & 104 & 4524 \\
\hline 11 & 31635 & 5128 & 1520 & 18654 & 22 & 651 & 1540 & 41 & 1906 \\
\hline
\end{tabular}

\subsection{Results}

In this section, we present computational results. The results were obtained using GAMS/Cplex on $2.3 \mathrm{GHz}$ Laptop computer with $4 \mathrm{~GB}$ of RAM under Windows 7. To solve the multi-objective model with $\varepsilon$-constraint method, first objective function is regarded as the most important objective function that it aims to minimize unsatisfied demand. The total cost of the pre-disaster phase is approximately 9 milliard dollars. The expected value of total cost for the post-disaster phase in this solution is about 23 milliard dollars. The expected value of the sum of maximum shortage for all AAs in this solution is 5.5 million units. Table 4 shows that nine RDCs are opened and distributed widely across the network. Figure 3 graphically shows the total cost as a function of the possible number of opened RDCs. According to Figure 3, the best value of total cost is obtained when number of RDCs is equal to 9, and 10.

Table 4: Amount of storage relief commodities in RDCs ( $10^{5}$ units)

\begin{tabular}{cccc}
\hline RDC & Size & Con. & Non-Con. \\
\hline 6 & Large & 7.7538 & 1.8 \\
\hline 7 & Large & 7.7538 & 1.8 \\
\hline 8 & Large & 7.7538 & 1.8 \\
\hline 9 & Medium & 5.2308 & 1.1667 \\
\hline 13 & Large & 7.7538 & 1.8 \\
\hline 14 & Small & 3.2308 & 0.75 \\
\hline 16 & Medium & 5.2308 & 1.1667 \\
\hline 18 & Medium & 5.2308 & 1.1667 \\
\hline 19 & Medium & 5.2308 & 1.1667 \\
\hline
\end{tabular}

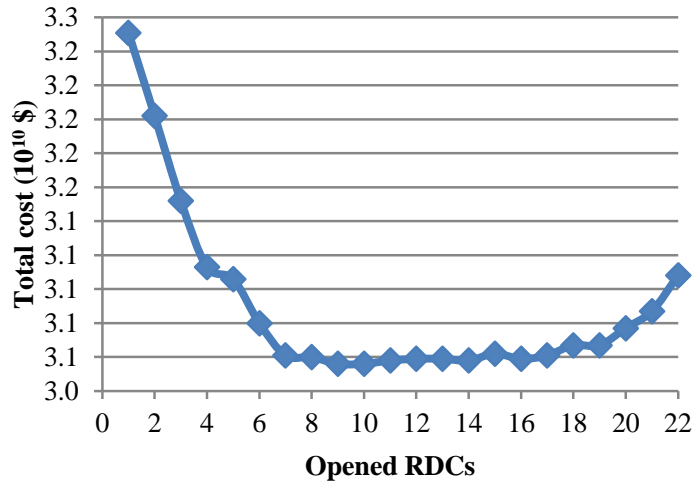

Figure 3: Total cost versus the number of selected RDCs

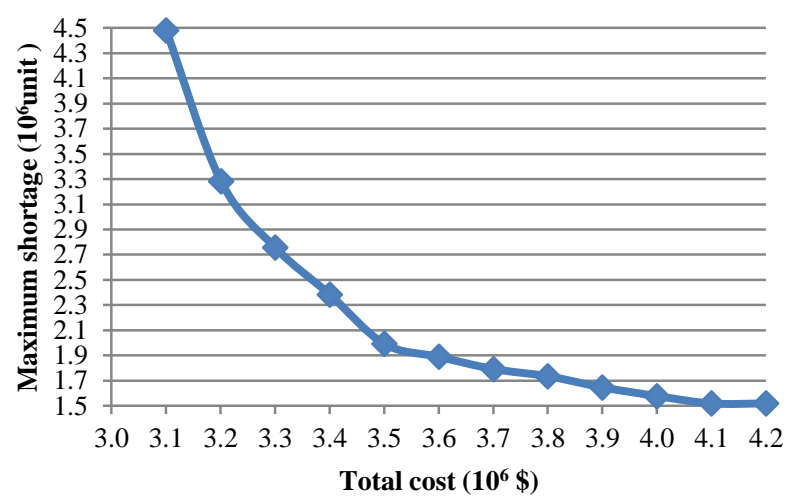

Figure 4: Efficient frontier for the maximum shortages against total cost 
To show the conflict there exists between the objective functions, the efficient frontiers are given in Figure 4. As can be seen, there is a significant conflict between total cost of relief chain and satisfaction level. As the unsatisfied demand increases, the total cost is reduced. Therefore, it is obvious that there are tradeoff situations for users to determine what decrements of the maximum shortage are desired.

In any case, the choice of the penalty cost parameters remains an important task for the decision maker. Figure 5 shows satisfaction level resulting from using different penalty cost parameters of 2, 5, 10, and 20 times its procurement cost for the four scenarios. As can be seen the considerable increase in satisfaction level can be achieved through the increase of penalty costs (except for the MF scenario where the demand is lower than in other scenarios). As the analysis shows, the appropriate choice also depends on the available budget: if the budget is not tight, higher levels of penalty costs are preferable in order to maximum satisfaction level.

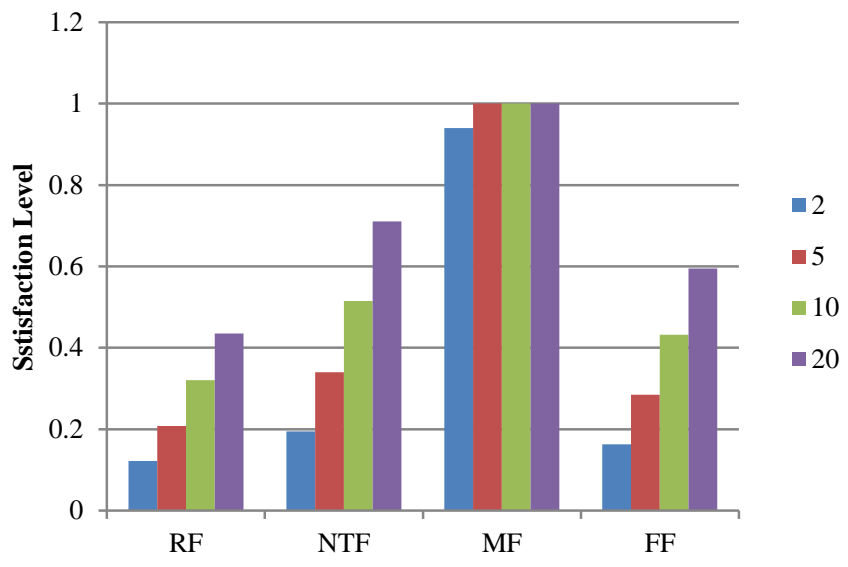

Figure 5: Satisfaction level with increased penalty for unmet demand

\section{Conclusions and Future Directions}

In this paper, we propose a new humanitarian relief logistics model which helps to build up an optimal predisaster plan while considering the post-disaster decisions. We formulated the problem as a multi-objective stochastic nonlinear program. The model helps to determine the locations of RDCs, the relief item inventory at the RDCs, the assignment and amount of relief item flows between the RDCs and demand points, and the amount of unsatisfied demand also called shortage corresponding to each demand point, item, scenario, and day. The proposed model highlighted two significant issues of supply chain as the objective; customer satisfaction and total cost. In our model, the cost parameters as well as demand and supply are subject to uncertainty. Furthermore, the model considers uncertainty in the locations where those demands might arise as well as the possibility that some of the pre-positioned supplies at RDC might be partially destroyed by the disaster. Uncertainty is represented by a set of discrete scenarios. Finally, model transformed into a linear one then multi-objective model was solved as a single- objective problem by applying $\varepsilon$-constraint method. To demonstrate the effectiveness of the proposed model, a case study based on a specific disaster scenario is presented. The interaction between the design objectives has been shown. This way of generating different possible configurations will help the decision-maker determine the best design according to the selected objectives. Sensitivity analysis is also performed to validate the model.

A number of extensions are possible for this model: (1) Aggregating this model with other assumption such as routing can be an interesting development. (2) For larger problems, solution times and hence computational 
burden of model analysis would increase. Therefore, future research would include the development of heuristic approaches that find near-optimal solutions for this model.

\section{References}

[1] http://www.emdat.com

[2] A. Afshar, and A. Haghani, Modeling integrated supply chain logistics in real-time large-scale disaster relief operations, Socio-Economic Planning Sciences, Article in press, pp.1-12 (2012).

[3] Y.H. Lin, R. Batta, P. Rogerson, A. Blatt, , and M. Flanigan, A logistics model for emergency supply of critical items in the aftermath of a disaster, Socio-Economic Planning Sciences, Vol. 45, No. 4, pp. 32-45 (2011).

[4] G.H. Tzeng, HJ. Cheng and TD. Huang, Multi-objective optimal planning for designing relief delivery systems, Transportation Research Part E, Vol.43, No. 6, pp. 673-686 (2007).

[5] B. Balcik and B.M. Beamon, Facility location in humanitarian relief, Journal of Logistics: Research and Applications,Vol. 11, No. 2, pp 101-121 (2008).

[6] G. Barbarosoglu and Y. Arda, A two-stage stochastic programming framework for transportation planning in disaster response, Journal of the Operational Research Society, Vol.55, pp 43-53 (2004).

[7] A. Bozorgi-Amiri, S. Jabalameli and S.M.J Mirzapour, A multi-objective robust stochastic programming model for disaster relief logistics under uncertainty, OR Spectrum, DOI: 10.1007/s00291-011-0268-x (2012).

[8] M.S. Chang, Y.L. Tseng, and J.W .Chen, A scenario planning approach for the flood emergency logistics preparation problem under uncertainty, Transportation Research Part E, Vol. 43, No. 6,pp. 737-754 (2007).

[9] H. Jia, F. Ordonez, and M. Dessouky, A modeling framework for facility location of medical services for large-scale emergencies, IIE Transactions, Vol. 39, No. 1, pp. 41-55 (2007).

[10] J. Salmeron and A. Apte, Stochastic optimization for natural disaster asset prepositioning, Production and Operations Management, Vol. 19, No. 5, pp. 561-574 (2010).

[11] O.N. Mete and Z. Zabinsky, Stochastic optimization of medical supply distribution, International Journal of Production Economics,Vol. 126, pp.76-84 (2010).

[12] C.G. Rawls, and M.A. Turnsquist, Pre-positioning of emergency supplies for disaster response,Transportation Research Part B,Vol. 44, No. 4, pp. 521-534 (2010).

[13] G. Mavrotas, Effective implementation of the $\varepsilon$-constraint method in Multi-Objective Mathematical Programming problems, Application Math Computer, Vol. 213, No. 2, pp. 455-465 (2009).

[14] M. Ashtari, D. Hatzfeld and N. Kamalian, Microseismicity in the region of Tehran, Tectonophysics, Vol. 395, No. 3, pp. 193-208 (2005). 